\title{
BALANCE FOR TATE COHOMOLOGY WITH RESPECT TO SEMIDUALIZING MODULES
}

\author{
LI LIANG ${ }^{\otimes}$ and GANG YANG \\ (Received 19 June 2012; accepted 4 March 2013; first published online 18 July 2013) \\ Communicated by B. Gardner
}

\begin{abstract}
In this paper, we further study Tate cohomology of modules over a commutative ring with respect to semidualizing modules using the ideals of Sather-Wagstaff et al. ['Tate cohomology with respect to semidualizing modules', J. Algebra 324 (2010), 2336-2368]. In particular, we prove a balance result for the Tate cohomology $\widehat{\mathrm{Ext}}^{n}$ for any $n \in \mathbb{Z}$. This result complements the work of Sather-Wagstaff et al., who proved that the result holds for any $n \geq 1$. We also discuss some vanishing properties of Tate cohomology.
\end{abstract}

2010 Mathematics subject classification: primary 13D07; secondary 18G15, 18G25, 13D02, 13D05, 18G10, $18 \mathrm{G} 20$.

Keywords and phrases: Tate cohomology, relative cohomology, balance, semidualizing modules.

\section{Introduction}

Tate cohomology was initially defined for representations of finite groups. Avramov and Martsinkovsky [1] extended the definition so that it can work well for finite modules of finite G-dimension over a Noetherian ring. They showed that if $M$ is a finite $R$-module of finite G-dimension, then there is an exact sequence connecting the absolute cohomology functor $\operatorname{Ext}_{R}^{*}(M,-)$, the relative cohomology functor $\operatorname{Ext}_{\mathcal{G}}^{*}(M,-)$ (that are defined by a proper Gorenstein projective resolution of $M$ ), and the Tate cohomology functor $\widehat{\operatorname{Ext}}_{R}^{*}(M,-)$ (see [1, (7.1)]).

Balancedness of absolute cohomology Ext $_{R}$ is well known. Holm [5, (3.6)] gave a balance result for the relative cohomlogy $\operatorname{Ext}_{\mathcal{G}}$ by showing that if $M$ is an $R$-module of finite Gorenstein projective dimension and $N$ is an $R$-module of finite Gorenstein injective dimension then $\operatorname{Ext}_{\mathcal{G}}^{*}(M, N)$ can also be computed using a proper Gorenstein injective resolution of $N$. Iacob [6, Theorem 2] proved a balance result for Tate

This work was partially supported by NSF of China (Grant Nos. 11226059, 11101197), the Young Scholars Science Foundation of Lanzhou Jiaotong University (Grant No. 2012020), and NSF of Gansu Province of China (No. 1107RJZA233).

(C) 2013 Australian Mathematical Publishing Association Inc. 1446-7887/2013 \$16.00 
cohomology $\widehat{\operatorname{Ext}}_{R}$ over Gorenstein rings. Recently, Christensen and Jorgensen [3] used the idea of a pinched complex to prove a general balance result for Tate cohomology, while Enochs et al. [4] gave a new way of constructing homology groups associated with a double complex, and with this result gave a new and elementary proof of balancedness of Tate cohomology.

Let $\mathcal{X}$ denote a subcategory of an abelian category $\mathcal{A}$ and $\mathcal{G}(\mathcal{X})$ denote the subcategory of $\mathcal{A}$ with objects of the form $M \cong \operatorname{Ker}\left(\delta_{-1}^{X}\right)$ for some totally $\mathcal{X}$-acyclic complex $X$ (see Section 2.3). Sather-Wagstaff et al. [9] constructed a theory of Tate cohomology in abelian categories. They proved the following balance result (see [9, (6.1)]).

Theorem. Let $\mathcal{W}$ and $\mathcal{V}$ be subcategories of $\mathcal{A}$. Assume that $\mathcal{W} \perp \mathcal{W}$ and $\mathcal{V} \perp \mathcal{V}$ and $\mathcal{G}(\mathcal{W}) \perp \mathcal{V}$ and $\mathcal{W} \perp \mathcal{G}(\mathcal{V})$. Assume that $\mathcal{W}$ is closed under kernels of epimorphisms and direct summands, and that $\mathcal{V}$ is closed under cokernels of monomorphisms and direct summands. Assume also that $\operatorname{Ext}_{\mathcal{W} \mathcal{A}}^{\geq 1}(\operatorname{res} \widehat{\mathcal{W}}, \mathcal{V})=0=\operatorname{Ext}_{\mathcal{A V} \mathcal{V}}^{\geq 1}(\mathcal{W}$, cores $\widehat{\mathcal{V}})$. Then, for all $M \in \operatorname{res} \widehat{\mathcal{G}(\mathcal{W})}$, all $N \in \operatorname{cores} \widehat{\mathcal{G}(\mathcal{V})}$ and all $n \geq 1$,

$$
\widehat{\operatorname{Ext}}_{\mathcal{W A}}^{n}(M, N) \cong \widehat{\operatorname{Ext}}_{\mathcal{A V} V}^{n}(M, N) \text {. }
$$

We notice that when $\mathcal{W}$ is the subcategory of projective $R$-modules and $\mathcal{V}$ is the subcategory of injective $R$-modules, then the above theorem gives a balance result for Tate cohomology $\widehat{\operatorname{Ext}}_{R}^{n}$ for $n \geq 1$ over any associative ring $R$.

In this paper, we further study balancedness of Tate cohomology in abelian categories. We show that the result of Sather-Wagstaffet al. [9, (6.1)] is true for any $n \in \mathbb{Z}$ (see Corollary 3.10). More generally, we prove the following result (see Theorem 3.9).

Theorem A. Let $\mathcal{X}, \mathcal{Y}, \mathcal{W}$ and $\mathcal{V}$ be subcategories of $\mathcal{A}$. Assume that $\mathcal{X}$ and $\mathcal{Y}$ are exact, and $\mathcal{X}$ is closed under kernels of epimorphisms and $\mathcal{Y}$ is closed under cokernels of monomorphisms. Assume that $\mathcal{W}$ is both an injective cogenerator and a projective generator for $\mathcal{X}$, and $\mathcal{V}$ is both an injective cogenerator and a projective generator for $\mathcal{Y}$. Assume also that $\mathcal{W}$ and $\mathcal{V}$ are closed under direct summands and satisfy $\mathcal{W} \perp \mathcal{Y}$, $\mathcal{X} \perp \mathcal{V}$ and $\operatorname{Ext}_{\mathcal{W} \mathcal{A}}^{\geq 1}(\operatorname{res} \widehat{\mathcal{W}}, \mathcal{V})=0=\operatorname{Ext}_{\mathcal{A} \mathcal{V}}^{\geq 1}(\mathcal{W}$, cores $\widehat{\mathcal{V}})$. Then, for all $M \in \operatorname{res} \widehat{\mathcal{X}}$ and $N \in \operatorname{cores} \widehat{\mathcal{Y}}$, and all $n \in \mathbb{Z}$,

$$
\widehat{\operatorname{Ext}}_{\mathcal{W A}}^{n}(M, N) \cong \widehat{\operatorname{Ext}}_{\mathcal{A} \mathcal{V}}^{n}(M, N) \text {. }
$$

As an application of Theorem A, we get the next balance result for Tate cohomology of modules with respect to semidualizing modules (see Corollary 3.12). This result was proved for each $n \geq 1$ in [9, Theorem D].

Theorem B. Let $R$ be a commutative ring, and let $B$ and $C$ be semidualizing $R$-modules such that $B \in G \mathcal{P}_{C}(R)$. Set $B^{\dagger}=\operatorname{Hom}_{R}(B, C)$. Let $M$ and $N$ be R-modules such that $\mathcal{G}\left(\mathcal{P}_{B}\right)-\operatorname{pd}_{R}(M)<\infty$ and $\mathcal{G}\left(\mathcal{I}_{B^{\dagger}}\right)-\mathrm{id}_{R}(N)<\infty$. Then, for each $n \in \mathbb{Z}$,

$$
\widehat{\operatorname{Ext}}_{\mathcal{P}_{B} \mathcal{M}}^{n}(M, N) \cong \widehat{\operatorname{Ext}}_{\mathcal{M} I_{B^{\dagger}}}^{n}(M, N) \text {. }
$$


Furthermore, under the hypothesis of Theorem B, we get that $M$ has a proper $\mathcal{P}_{\mathcal{B}}$-resolution $W \stackrel{\simeq}{\longrightarrow} M$ and a proper $\mathcal{G}\left(\mathcal{P}_{B}\right)$-resolution $X \stackrel{\simeq}{\longrightarrow} M$ by Lemma 2.6. Set $\overline{\mathrm{id}_{M}}: W \rightarrow X$ to be a lifting of the identity $\operatorname{id}_{M}: M \rightarrow M$. Dually, one can construct $\overline{\mathrm{id}_{N}}$. Then the next result provides a new method to compute Tate cohomology of modules with respect to semidualizing modules (see Corollary 3.16).

Theorem C. Let $R$ be a commutative ring, and let $B$ and $C$ be semidualizing $R$-modules such that $B \in G^{P} P_{C}(R)$. Set $B^{\dagger}=\operatorname{Hom}_{R}(B, C)$. Let $M$ and $N$ be R-modules such that $\mathcal{G}\left(\mathcal{P}_{B}\right)-\operatorname{pd}_{R}(M)<\infty$ and $\mathcal{G}\left(\mathcal{I}_{B^{\dagger}}\right)-\mathrm{id}_{R}(N)<\infty$. Then, for each $n \geq 1$,

$$
\begin{aligned}
\widehat{\operatorname{Ext}}_{\mathcal{P}_{B} \mathcal{M}}^{n}(M, N) & \cong \widehat{\operatorname{Ext}}_{\mathcal{M} I_{B^{\dagger}}}^{n}(M, N) \\
& \cong \mathrm{H}_{-n-1}\left(\operatorname{Hom}_{R}\left(\operatorname{Cone}\left(\overline{\mathrm{id}_{M}}\right), N\right)\right) \\
& \cong \mathrm{H}_{-n}\left(\operatorname{Hom}_{R}\left(M, \operatorname{Cone}\left(\overline{\operatorname{id}_{N}}\right)\right)\right)
\end{aligned}
$$

As we will see, the vanishing properties of Tate cohomology play an important role in the proof of Theorem A. We prove the next vanishing result that encompasses the results of Sather-Wagstaff et al. [9, (5.2), (5.6) and (5.7)] (see Theorem 3.5 and Corollary 3.6).

Theorem D. Let $\mathcal{X}$ and $\mathcal{W}$ be subcategories of $\mathcal{A}$. Assume that $\mathcal{X}$ is exact and closed under kernels of epimorphisms, and that $\mathcal{W}$ is both an injective cogenerator and a projective generator for $\mathcal{X}$ and closed under direct summands. If $M \in \operatorname{res} \widehat{\mathcal{X}}$, then the following statements are equivalent.

(1) $M \in \operatorname{res} \widehat{\mathcal{W}}$.

(2) $\widehat{\operatorname{Ext}}_{W \mathcal{A}}^{n}(-, M)=0$ on res $\widehat{X}$ for each $n \in \mathbb{Z}$.

(3) $\widehat{\operatorname{Ext}}_{W \mathcal{A}}^{n}(M,-)=0$ for each $n \in \mathbb{Z}$.

(4) $\widehat{\operatorname{Ext}}_{W \mathcal{A}}^{n}(-, M)=0$ on res $\widehat{\mathcal{X}}$ for some $n \in \mathbb{Z}$.

(5) $\widehat{\operatorname{Ext}}_{\mathcal{W A}}^{n}(M,-)=0$ for some $n \in \mathbb{Z}$.

(6) $\widehat{\operatorname{Ext}}_{W \mathcal{A}}(M, M)=0$.

(7) The transformation $\vartheta_{X \mathcal{W A}}^{i}(-, M): \operatorname{Ext}_{\mathcal{X A}}^{i}(-, M) \rightarrow \operatorname{Ext}_{\mathcal{W A}}^{i}(-, M)$ is an isomorphism on res $\widehat{X}$ for each $i \in \mathbb{Z}$.

(8) The transformation $\vartheta_{\mathcal{X W \mathcal { A }}}^{i}(M,-): \operatorname{Ext}_{\mathcal{X} \mathcal{A}}^{i}(M,-) \rightarrow \operatorname{Ext}_{\mathcal{W A}}^{i}(M,-)$ is an isomorphism for each $i \in \mathbb{Z}$.

(9) The transformation $\vartheta_{\mathcal{X W \mathcal { A }}}^{i}(-, M): \operatorname{Ext}_{\mathcal{X A}}^{i}(-, M) \rightarrow \operatorname{Ext}_{\mathcal{W A}}^{i}(-, M)$ is an isomorphism on res $\widehat{X}$ for each $1 \leq i \leq 2$.

(10) The transformation $\vartheta_{\mathcal{X W \mathcal { A }}}^{i}(M,-): \operatorname{Ext}_{\mathcal{X} \mathcal{A}}^{i}(M,-) \rightarrow \operatorname{Ext}_{\mathcal{W A}}^{i}(M,-)$ is an isomorphism either for two successive values of $i$ with $1 \leq i<d$ or for a single value of $i$ with $i \geq d$, where $d=\mathcal{X}-\operatorname{pd}(M)<\infty$.

The dual result is given in Theorem 3.7 and Corollary 3.8. 


\section{Preliminaries}

We begin with some notation and terminology for use throughout this paper.

2.1. Throughout this work, $\mathcal{A}$ always denotes an abelian category, and given a ring $R$, $\mathcal{M}$ denotes the category of left $R$-modules. We use the term 'subcategory' for a 'full additive subcategory' that is closed under isomorphisms. A subcategory $\mathcal{X}$ of $\mathcal{A}$ is exact if it is closed under direct summands and extensions.

We fix subcategories $\mathcal{X}, \mathcal{Y}, \mathcal{W}$ and $\mathcal{V}$ of $\mathcal{A}$ such that $\mathcal{W} \subseteq \mathcal{X}$ and $\mathcal{V} \subseteq \mathcal{Y}$. Write $\mathcal{X} \perp \mathcal{Y}$ if $\operatorname{Ext}_{\mathcal{A}}^{\geq 1}(X, Y)=0$, and $\mathcal{X} \perp_{1} \mathcal{Y}$ if $\operatorname{Ext}_{\mathcal{A}}^{1}(X, Y)=0$ for any $X \in \mathcal{X}$ and any $Y \in \mathcal{Y}$. For an object $M$ of $\mathcal{A}$, write $M \perp_{1} \mathcal{Y}$ (respectively, $\left.X \perp_{1} M\right)$ if $\operatorname{Ext}_{\mathcal{A}}^{1}(M, Y)=0$ for any $Y \in \mathcal{Y}$ (respectively, if $\operatorname{Ext}_{\mathcal{A}}^{1}(X, M)=0$ for any $\left.X \in \mathcal{X}\right)$. We say that $\mathcal{W}$ is a generator for $X$ if, for any $X \in X$, there is an exact sequence $0 \rightarrow X^{\prime} \rightarrow W \rightarrow X \rightarrow 0$ such that $W \in \mathcal{W}$ and $X^{\prime} \in \mathcal{X}$. The subcategory $\mathcal{W}$ is a projective generator for $\mathcal{X}$ if $\mathcal{W}$ is a generator for $\mathcal{X}$ and $\mathcal{W} \perp \mathcal{X}$. Dually, one can give the concepts of cogenerators and injective cogenerators.

2.2. A complex $\cdots \longrightarrow X_{1} \stackrel{\delta_{1}^{X}}{\longrightarrow} X_{0} \stackrel{\delta_{0}^{X}}{\longrightarrow} X_{-1} \longrightarrow \cdots$ of objects of $\mathcal{A}$ will be denoted by $\left(X, \delta^{X}\right)$ or simply $X$. We frequently (and without warning) identify objects of $\mathcal{A}$ with complexes concentrated in degree zero. A complex $X$ is bounded above if $X_{n}=0$ for $n \gg 0$, and it is bounded below if $X_{n}=0$ for $n \ll 0$. A complex $X$ is bounded if it is both bounded above and bounded below. The $n$th homology of $X$ is defined as $\operatorname{Ker} \delta_{n}^{X} / \operatorname{Im} \delta_{n+1}^{X}$ and it is denoted by $\mathrm{H}_{n}(X)$. For any $m \in \mathbb{Z}, \Sigma^{m} X$ denotes the complex with the degree- $n$ term $\left(\Sigma^{m} X\right)_{n}=X_{n-m}$ and whose boundary operators are $(-1)^{m} \delta_{n-m}^{X}$. We set $\Sigma M=\Sigma^{1} M$. The soft truncations of $X$ at $n$ are the complexes

$$
X_{\subset n} \equiv 0 \longrightarrow \operatorname{Coker}\left(\delta_{n+1}^{X}\right) \stackrel{\overline{\delta_{n}^{X}}}{\longrightarrow} X_{n-1} \stackrel{\delta_{n-1}^{X}}{\longrightarrow} X_{n-2} \longrightarrow \cdots
$$

and

$$
X_{\supset n} \equiv \cdots \longrightarrow X_{n+2} \stackrel{\delta_{n+2}^{X}}{\longrightarrow} X_{n+1} \stackrel{\delta_{n+1}^{X}}{\longrightarrow} \operatorname{Ker}\left(\delta_{n}^{X}\right) \longrightarrow 0
$$

If $X$ and $Y$ are both complexes, then by a morphism $\alpha: X \rightarrow Y$ we mean a sequence $\alpha_{n}: X_{n} \rightarrow Y_{n}$ such that $\alpha_{n-1} \delta_{n}^{X}=\delta_{n}^{Y} \alpha_{n}$ for each $n \in \mathbb{Z}$. A quasiisomorphism, indicated by the symbol ' $\simeq$ ', is a morphism of complexes that induces an isomorphism in homology. The mapping cone Cone $(\alpha)$ of $\alpha$ is defined as Cone $(\alpha)_{n}=Y_{n} \oplus X_{n-1}$ with $n$th boundary operator $\delta_{n}^{\operatorname{Cone}(\alpha)}=\left(\begin{array}{cc}\delta_{n}^{Y} & \alpha_{n-1} \\ 0 & -\delta_{n-1}^{X}\end{array}\right)$. It is well known that a morphism $\alpha$ is a quasiisomorphism if and only if its mapping cone Cone $(\alpha)$ is exact. The Hom-complex $\operatorname{Hom}_{\mathcal{A}}(X, Y)$ denotes the complex of abelian groups with the degree- $n$ term $\operatorname{Hom}_{\mathcal{A}}(X, Y)_{n}=\prod_{t \in \mathbb{Z}} \operatorname{Hom}_{\mathcal{A}}\left(X_{t}, Y_{n+t}\right)$ and whose $n$th boundary operator is given by $\left\{f_{t}\right\} \mapsto\left\{\delta_{t+n}^{Y} f_{t}-(-1)^{n} f_{t-1} \delta_{t}^{X}\right\}$. One can check that a morphism from $X$ to $Y$ is an element of $\operatorname{Ker}\left(\delta_{0}^{\operatorname{Hom}_{\mathcal{A}}(X, Y)}\right)$. A complex $T$ is $\operatorname{Hom}_{\mathcal{A}}(X,-)$-exact if $\operatorname{Hom}_{\mathcal{A}}(M, T)$ is exact for each object $M \in \mathcal{X}$. The term $\operatorname{Hom}_{\mathcal{A}}(-, \mathcal{X})$-exact is defined dually. 
2.3. An exact complex of objects in $\mathcal{X}$ is totally $\mathcal{X}$-acyclic if it is $\operatorname{Hom}_{\mathcal{A}}(\mathcal{X},-)$-exact and $\operatorname{Hom}_{\mathcal{A}}(-, \mathcal{X})$-exact. Let $\mathcal{G}(\mathcal{X})$ denote the subcategory of $\mathcal{A}$ with objects of the form $M \cong \operatorname{Ker}\left(\delta_{-1}^{X}\right)$ for some totally $\mathcal{X}$-acyclic complex $X$.

Remark 2.4. If $\mathcal{W} \perp \mathcal{W}$, then, by [7, Theorem B and Corollary 4.7], $\mathcal{W}$ is both an injective cogenerator and a projective generator for $\mathcal{G}(\mathcal{W})$, and $\mathcal{G}(\mathcal{W})$ is an exact subcategory of $\mathcal{A}$, and it is closed under kernels of epimorphisms (or cokernels of monomorphisms) if $\mathcal{W}$ is.

One can find the following definitions in [9].

2.5. Let $M$ be an object of $\mathcal{A}$. An $\mathcal{X}$-resolution of $M$ is a complex $X$ of objects in $\mathcal{X}$ such that $X_{-n}=0=\mathrm{H}_{n}(X)$ for all $n>0$ and $\mathrm{H}_{0}(X) \cong M$. The associated exact sequence

$$
X^{+} \equiv \cdots \rightarrow X_{1} \rightarrow X_{0} \rightarrow M \rightarrow 0
$$

is the augmented $\mathcal{X}$-resolution of $M$ associated to $X$. Sometimes we call the quasiisomorphism $X \stackrel{\simeq}{\longrightarrow} M$ an $\mathcal{X}$-resolution of $M$. A bounded strict $\mathcal{W} \mathcal{X}$-resolution

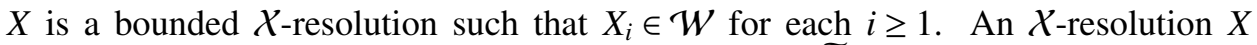
is proper if $X^{+}$is $\operatorname{Hom}_{\mathcal{A}}(X,-)$-exact, and we let res $\widetilde{X}$ denote the subcategory of objects of $\mathcal{A}$ admitting a proper $\mathcal{X}$-resolution. The $\mathcal{X}$-projective dimension of $M$ is the quantity

$$
\mathcal{X}-\operatorname{pd}(M)=\inf \left\{\sup \left\{n \geq 0 \mid X_{n} \neq 0\right\} \mid X \text { is an } \mathcal{X} \text {-resolution of } M\right\} .
$$

We let res $\widehat{X}$ denote the subcategory of objects of $\mathcal{A}$ of finite $\mathcal{X}$-projective dimension.

We define (proper) $\mathcal{Y}$-coresolutions and $\mathcal{Y}$-injective dimension, $\mathcal{Y}$ - $\operatorname{id}(M)$, of $M$ dually. We let cores $\widetilde{\mathcal{Y}}$ and cores $\widehat{\mathcal{Y}}$ denote the subcategories of objects of $\mathcal{A}$ admitting a proper $\mathcal{Y}$-coresolution and objects of $\mathcal{A}$ of finite $\mathcal{Y}$-injective dimension, respectively. Similarly, a bounded strict $\mathcal{Y} \mathcal{V}$-coresolution $Y$ of $M$ is a bounded $\mathcal{Y}$-coresolution such that $Y_{i} \in \mathcal{V}$ for $i \leq-1$.

By $[8,(3.3)$ and (3.4)], we have the following result.

Lemma 2.6. Assume that $\mathcal{X}$ and $\mathcal{Y}$ are closed under extensions. Assume that $\mathcal{W}$ is both an injective cogenerator and a projective generator for $\mathcal{X}$, and that $\mathcal{V}$ is both an injective cogenerator and a projective generator for $\mathcal{Y}$. Then $\operatorname{res} \widehat{\mathcal{X}} \subseteq \operatorname{res} \widetilde{\mathcal{W}} \cap \operatorname{res} \widetilde{\mathcal{X}}$ and cores $\widehat{\mathcal{Y}} \subseteq \operatorname{cores} \widetilde{\mathcal{V}} \cap \operatorname{cores} \widetilde{\mathcal{Y}}$.

2.7. Let $M$ and $N$ be objects of $\mathcal{A}$. If $M$ admits a proper $\mathcal{X}$-resolution $X \stackrel{\simeq}{\longrightarrow} M$, then the $n$th relative cohomology group $\operatorname{Ext}_{\mathcal{X} \mathcal{A}}^{n}(M, N)$ is

$$
\operatorname{Ext}_{\mathcal{X} \mathcal{A}}^{n}(M, N)=\mathrm{H}_{-n}\left(\operatorname{Hom}_{\mathcal{A}}(X, N)\right)
$$


If $N$ admits a proper $\mathcal{Y}$-coresolution, the $n$th relative cohomology group $\operatorname{Ext}_{\mathcal{A} y}^{n}(M, N)$ is defined dually.

Assume that $M$ admits a proper $\mathcal{W}$-resolution $W \stackrel{\gamma}{\rightarrow} M$ and a proper $\mathcal{X}$-resolution $X \stackrel{\gamma^{\prime}}{\rightarrow} M$. Let $\overline{\mathrm{id}_{M}}: W \rightarrow X$ be a lifting of the identity $\operatorname{id}_{M}: M \rightarrow M$, then $\overline{\mathrm{id}_{M}}$ is a quasiisomorphism such that $\gamma=\gamma^{\prime} \circ \overline{\mathrm{id}_{M}}$. We set

$$
\vartheta_{\mathcal{X} \mathcal{F}}^{n}(M,-)=\mathrm{H}_{-n}\left(\operatorname{Hom}_{\mathcal{A}}\left(\overline{\operatorname{id}_{M}},-\right)\right): \operatorname{Ext}_{\mathcal{X} \mathcal{A}}^{n}(M,-) \rightarrow \operatorname{Ext}_{\mathcal{W A}}^{n}(M,-)
$$

When $N$ admits a proper $\mathcal{V}$-coresolution and a proper $\mathcal{Y}$-coresolution, the map

$$
\vartheta_{\mathcal{A Y} \mathcal{V}}^{n}(-, N): \operatorname{Ext}_{\mathcal{A} \mathcal{Y}}^{n}(-, N) \rightarrow \operatorname{Ext}_{\mathcal{A V} \mathcal{V}}^{n}(-, N)
$$

is defined dually.

2.8. Let $M$ and $N$ be objects of $\mathcal{A}$. A Tate $\mathcal{W}$-resolution of $M$ is a diagram $T \stackrel{\alpha}{\rightarrow} W \stackrel{\gamma}{\rightarrow} M$ of morphisms of complexes, where $T$ is a totally $\mathcal{W}$-acyclic complex, $\gamma$ is a proper $\mathcal{W}$-resolution of $M$, and $\alpha_{n}$ is an isomorphism for $n \gg 0$. We let res $\bar{W}$ denote the subcategory of objects of $\mathcal{A}$ admitting a Tate $\mathcal{W}$-resolution. A Tate $\mathcal{V}$-coresolution of $N$ is defined dually, and we let cores $\overline{\mathcal{V}}$ denote the subcategory of objects of $\mathcal{A}$ admitting a Tate $\mathcal{V}$-coresolution. Then res $\overline{\mathcal{W}}$ and cores $\overline{\mathcal{V}}$ are subcategories of $\mathcal{A}$, and $\mathcal{G}(\mathcal{W}) \subseteq \operatorname{res} \overline{\mathcal{W}} \subseteq \operatorname{res} \widetilde{\mathcal{W}}$ and $\mathcal{G}(\mathcal{V}) \subseteq \operatorname{cores} \overline{\mathcal{V}} \subseteq \operatorname{cores} \widetilde{\mathcal{V}}$. If $\mathcal{W} \perp \mathcal{W}$, then res $\widehat{\mathcal{W}} \subseteq \operatorname{res} \overline{\mathcal{W}}$ and cores $\widehat{\mathcal{W}} \subseteq \operatorname{cores} \overline{\mathcal{W}}$ (see [9, (3.2)]).

If $M$ admits a Tate $W$-resolution $T \rightarrow W \rightarrow M$, define the $n$th Tate cohomology group $\widehat{\operatorname{Ext}}_{\mathcal{W A}}^{n}(M, N)$ as

$$
\widehat{\operatorname{Ext}}_{\mathcal{W A}}^{n}(M, N)=\mathrm{H}_{-n}\left(\operatorname{Hom}_{\mathcal{A}}(T, N)\right)
$$

for each $n \in \mathbb{Z}$. It follows from [9, (3.8)] that this definition is independent (up to isomorphism) of the choice of Tate $\mathcal{W}$-resolution. Dually, if $N$ admits a Tate $\mathcal{V}$ coresolution $N \rightarrow V \rightarrow S$, define the $n$th Tate cohomology group $\widehat{\operatorname{Ext}}_{\mathcal{A V}}^{n}(M, N)$ as

$$
\widehat{\operatorname{Ext}}_{\mathcal{A} \mathcal{V}}^{n}(M, N)=\mathrm{H}_{-n}\left(\operatorname{Hom}_{\mathcal{A}}(M, S)\right)
$$

for each $n \in \mathbb{Z}$. This definition is also independent (up to isomorphism) of the choice of Tate $\mathcal{V}$-coresolution by [9, (3.8)].

\section{Tate cohomology in Abelian categories}

We begin with the following lemmas that are tools for the proof of Theorem 3.5.

Lemma $3.1[9,(4.5)]$. Assume that $\mathcal{W} \perp \mathcal{W}$ and $\mathcal{V} \perp \mathcal{V}$. Let $M$ and $N$ be objects of $\mathcal{A}$, then the following statements hold. 
(1) If $M \in \operatorname{res} \widehat{\mathcal{W}}$, then $\widehat{\operatorname{Ext}}_{\mathcal{W A}}^{n}(-, M)=0$ on $\operatorname{res} \overline{\mathcal{W}}$ and $\widehat{\operatorname{Ext}}_{\mathcal{W A}}^{n}(M,-)=0$ for all $n \in \mathbb{Z}$.

(2) If $N \in \operatorname{cores} \widehat{\mathcal{V}}$, then $\widehat{\operatorname{Ext}}_{\mathcal{A V} \mathcal{V}}^{n}(N,-)=0$ on cores $\overline{\mathcal{V}}$ and $\widehat{\operatorname{Ext}}_{\mathcal{A V} \mathcal{V}}^{n}(-, N)=0$ for all $n \in \mathbb{Z}$.

Lemma 3.2. Assume that $\mathcal{W}$ is closed under direct summands and $\mathcal{W} \perp \mathcal{W}$, and let $M \in \operatorname{res} \bar{W}$. If $\widehat{\operatorname{Ext}}_{\mathcal{W A}}^{0}(M, M)=0$ or $\widehat{\operatorname{Ext}}_{\mathcal{A W}}^{0}(M, M)=0$, then $M \in \operatorname{res} \widehat{\mathcal{W}}$.

Proof. We prove the case when $\widehat{\operatorname{Ext}}_{W \mathcal{A}}^{0}(M, M)=0$; the proof of the other case is dual. Since $M \in \operatorname{res} \bar{W}$, without loss of generality, we may assume that there is a Tate $\mathcal{W}$ resolution $T \stackrel{\alpha}{\rightarrow} W \stackrel{\gamma}{\rightarrow} M$ of $M$ such that $\alpha_{n}$ is an isomorphism for each $n \geq t$, where $t \geq 1$. Let $M_{i}=\operatorname{Im}\left(\delta_{i}^{W}\right)$ for $i \geq 1$, then $M_{i} \in \operatorname{res} \bar{W}$. Note that the exact sequence

$$
\cdots \rightarrow W_{t} \rightarrow \cdots \rightarrow W_{0} \stackrel{\gamma}{\rightarrow} M \rightarrow 0
$$

is $\operatorname{Hom}_{\mathcal{A}}(\mathcal{W},-)$-exact and $W_{i} \in \mathcal{W}$ for $i \geq 0$, so

$$
\widehat{\operatorname{Ext}}_{W \mathcal{A}}^{j}\left(A, W_{i}\right)=0=\widehat{\operatorname{Ext}}_{W \mathcal{A}}^{j}\left(W_{i}, B\right)
$$

for any $j \in \mathbb{Z}$, any $i \geq 0$, any object $B$ of $\mathcal{A}$ and any $A \in \operatorname{res} \overline{\mathcal{W}}$ by Lemma 3.1. Thus, by [9, (4.6) and (4.7)],

$$
\widehat{\operatorname{Ext}}_{\mathcal{W A}}^{0}\left(M_{t}, M_{t}\right) \cong \widehat{\operatorname{Ext}}_{\mathcal{W A}}^{t}\left(M, M_{t}\right) \cong \widehat{\operatorname{Ext}}_{\mathcal{W A}}^{0}(M, M)=0
$$

Note that $M_{t} \in \mathcal{G}(\mathcal{W})$, so that $M_{t} \in \mathcal{W}$ by $[9,(5.1)]$, and hence $M \in \operatorname{res} \widehat{\mathcal{W}}$.

Lemma 3.3. Assume that $\mathcal{X}$ and $\mathcal{Y}$ are exact, $\mathcal{W}$ is a generator for $\mathcal{X}$ and $\mathcal{V}$ is $a$ cogenerator for $\mathcal{Y}$. Consider the exact sequence

$$
0 \rightarrow M^{\prime} \longrightarrow M \longrightarrow M^{\prime \prime} \longrightarrow 0
$$

of objects of $\mathcal{A}$, then the following statements hold.

(1) If $M^{\prime \prime}, M \in \mathcal{X}$ and $\mathcal{W} \perp_{1} M^{\prime}$, then $M^{\prime} \in \mathcal{X}$; if $\mathcal{W}$ is closed under direct summands, $M^{\prime}, M \in \mathcal{X}$ and $M^{\prime \prime} \perp_{1} \mathcal{X}$, then $M^{\prime \prime} \in \mathcal{W}$.

(2) If $M^{\prime}, M \in \mathcal{Y}$ and $M^{\prime \prime} \perp_{1} \mathcal{V}$, then $M^{\prime \prime} \in \mathcal{Y}$; if $\mathcal{V}$ is closed under direct summands, $M^{\prime \prime}, M \in \mathcal{Y}$ and $\mathcal{Y} \perp_{1} M^{\prime}$, then $M^{\prime} \in \mathcal{V}$.

Proof. We prove part (1); the proof of part (2) is dual. Since $M^{\prime \prime} \in \mathcal{X}$, there is an exact sequence

$$
0 \rightarrow X \rightarrow W \rightarrow M^{\prime \prime} \longrightarrow 0
$$


with $W \in \mathcal{W}$ and $X \in \mathcal{X}$. Consider the following pullback diagram.

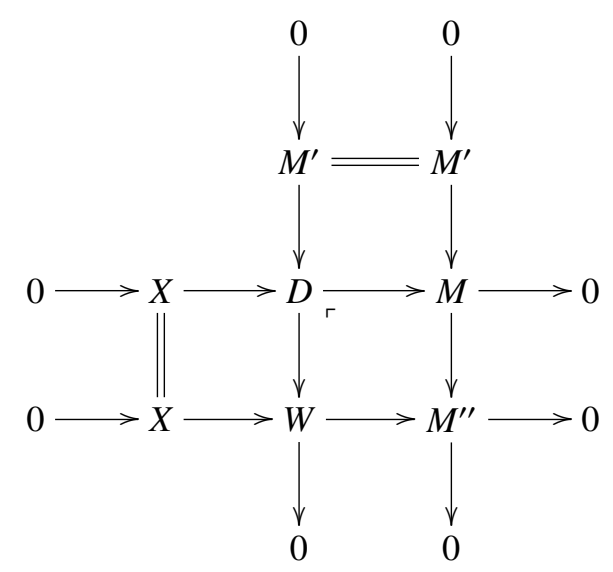

Since $M, X \in \mathcal{X}$, the exactness of the middle row, with the fact that $\mathcal{X}$ is closed under extensions, implies that $D \in \mathcal{X}$. Note that $\operatorname{Ext}_{\mathcal{A}}^{1}\left(W, M^{\prime}\right)=0$ since $W \in \mathcal{W}$, so the middle column is split, and hence $M^{\prime} \in \mathcal{X}$.

For the other part, since $M \in \mathcal{X}$, there is an exact sequence

$$
0 \rightarrow X \rightarrow W \rightarrow M \rightarrow 0
$$

with $W \in \mathcal{W}$ and $X \in \mathcal{X}$. Consider the following pullback diagram.

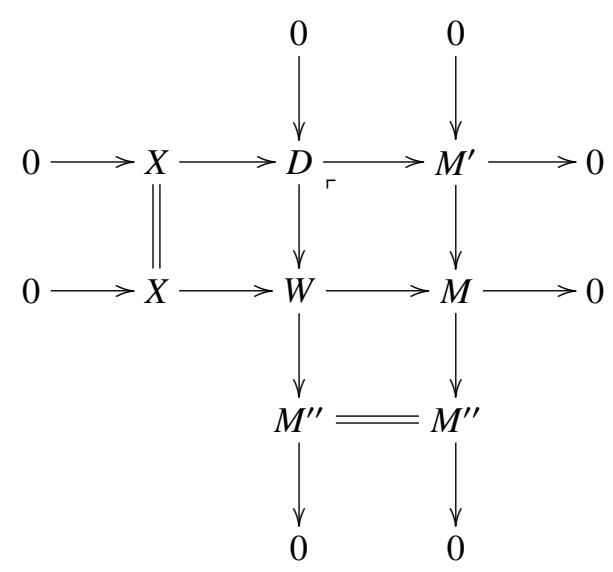

Since $M^{\prime}, X \in \mathcal{X}$, the exactness of the top row and the fact that $\mathcal{X}$ is closed under extensions imply that $D \in \mathcal{X}$, and hence $\operatorname{Ext}_{\mathcal{A}}^{1}\left(M^{\prime \prime}, D\right)=0$. Thus the middle column is split, and so $M^{\prime \prime} \in \mathcal{W}$ since $W \in \mathcal{W}$.

Lemma 3.4. Assume that $\mathcal{X}$ is exact and $\mathcal{W}$ is a projective generator for $\mathcal{X}$. Let $M \in \operatorname{res} \widehat{\mathcal{X}}$ with $\mathcal{X}-\operatorname{pd}(M)=t<\infty$. If $M \in \operatorname{res} \widetilde{\mathcal{W}}$ with $W \stackrel{\sim}{\longrightarrow} M$ a proper $\mathcal{W}$-resolution of $M$, then $K_{t}=\operatorname{Im}\left(W_{t} \rightarrow W_{t-1}\right) \in \mathcal{X}$ with $W_{-1}=M$. 
Proof. If $t=0$, then $K_{0}=M \in \mathcal{X}$. Let $t>0$, and let

$$
0 \rightarrow X_{t} \rightarrow \cdots \rightarrow X_{0} \rightarrow M \rightarrow 0
$$

be an augmented $\mathcal{X}$-resolution of $M$, then it is $\operatorname{Hom}_{\mathcal{A}}(\mathcal{W},-)$-exact since $\mathcal{W} \perp \mathcal{X}$. Thus we get the following commutative diagram.

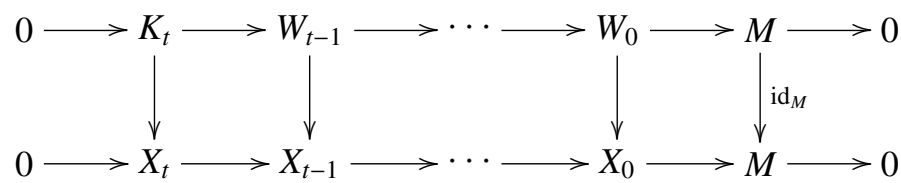

Since each row is exact and $\operatorname{Hom}_{\mathcal{A}}(\mathcal{W},-)$-exact, we get that the mapping cone

$$
0 \longrightarrow K_{t} \rightarrow X_{t} \oplus W_{t-1} \longrightarrow \cdots \rightarrow X_{1} \oplus W_{0} \rightarrow X_{0} \oplus M \longrightarrow M \longrightarrow 0
$$

is exact and $\operatorname{Hom}_{\mathcal{A}}(\mathcal{W},-)$-exact. Thus the sequence

$$
0 \longrightarrow K_{t} \rightarrow X_{t} \oplus W_{t-1} \longrightarrow \cdots \rightarrow X_{1} \oplus W_{0} \rightarrow X_{0} \rightarrow 0
$$

is exact and $\operatorname{Hom}_{\mathcal{A}}(\mathcal{W},-)$-exact. Now, repeated application of Lemma 3.3 yields $K_{t} \in \mathcal{X}$.

The next result encompasses [9, (5.2)]. Notice that, even when $\mathcal{X}$ is exact and closed under kernels of epimorphisms, and $\mathcal{W}$ is both an injective cogenerator and a projective generator for $\mathcal{X}$ and closed under direct summands, one may have $\mathcal{X} \mathcal{G}(\mathcal{W})($ see $[9,(3.12)])$.

Theorem 3.5. Assume that $\mathcal{X}$ is exact and closed under kernels of epimorphisms, and that $\mathcal{W}$ is both an injective cogenerator and a projective generator for $\mathcal{X}$ and closed under direct summands. Let $M \in \operatorname{res} \widehat{X}$. Then the following statements are equivalent:

(1) $M \in \operatorname{res} \widehat{\mathcal{W}}$;

(2) $\widehat{\operatorname{Ext}}_{W \mathcal{A}}^{n}(-, M)=0$ on res $\widehat{\mathcal{X}}$ for each $n \in \mathbb{Z}$;

(2') $\widehat{\operatorname{Ext}}_{W \mathcal{A}}^{n}(M,-)=0$ for each $n \in \mathbb{Z}$;

(3) $\widehat{\operatorname{Ext}}_{W \mathcal{A}}^{n}(-, M)=0$ on res $\widehat{X}$ for some $n \in \mathbb{Z}$;

(3') $\widehat{\operatorname{Ext}}_{W \mathcal{A}}^{n}(M,-)=0$ for some $n \in \mathbb{Z}$;

(4) $\widehat{\operatorname{Ext}}_{\mathcal{W A}}(M, M)=0$.

Proof. (1) $\Rightarrow$ (2) follows from Lemma 3.1 and [9, (3.4)].

$(2) \Rightarrow(3)$ is trivial.

(3) $\Rightarrow(4)$. Assume that $\widehat{\operatorname{Ext}}_{W \mathcal{A}}^{n}(-, M)=0$ on res $\widehat{X}$ for some $n \in \mathbb{Z}$. If $n=0$, then the condition (4) holds immediately.

Let $n<0$, and let $n=-d$ with $d>0$. Since $M \in \operatorname{res} \widehat{X}$, we get $M \in \operatorname{res} \widetilde{W}$ by $[8$, (3.4)]. Let $W \stackrel{\simeq}{\longrightarrow} M$ be a proper $\mathcal{W}$-resolution of $M$, and let $M_{i} \in \operatorname{Im}\left(W_{i} \rightarrow W_{i-1}\right)$ 
for $i \geq 1$, then $M_{i} \in \operatorname{res} \widehat{X}$ by Lemmas 3.3(1) and 3.4. Note that, for any $t \in \mathbb{Z}$ and any $i \geq 0, \widehat{\operatorname{Ext}}_{W \mathcal{A}}^{t}\left(W_{i}, M\right)=0$ by Lemma 3.1 , so

$$
\widehat{\operatorname{Ext}}_{\mathcal{W A}}^{0}(M, M) \cong \widehat{\operatorname{Ext}}_{\mathcal{W A}}^{-d}\left(M_{d}, M\right)=0
$$

by $[9,(4.6)]$ since $M_{d} \in \operatorname{res} \widehat{X}$.

Let $n>0$. By [8, (3.3)], there is an exact sequence

$$
0 \rightarrow M \rightarrow W_{-1} \rightarrow M_{-1} \rightarrow 0
$$

with $W_{-1} \in \operatorname{res} \widehat{\mathcal{W}}$ and $M_{-1} \in \mathcal{X}$. Since $\mathcal{W} \perp \operatorname{res} \widehat{\mathcal{X}}$, the sequence $(*)$ is $\operatorname{Hom}_{\mathcal{A}}(\mathcal{W},-)$ exact. Note that $\mathcal{W}$ is an injective cogenerator for $\mathcal{X}$, so there is an exact sequence

$$
0 \rightarrow M_{-1} \rightarrow W_{-2} \rightarrow W_{-3} \rightarrow \cdots
$$

with $W_{i} \in \mathcal{W}$ for $i \leq-2$, such that $M_{-i}=\operatorname{Im}\left(W_{-i} \rightarrow W_{-i-1}\right) \in \mathcal{X}$ for $i \geq 2$. Obviously, the sequence (**) is $\operatorname{Hom}_{\mathcal{A}}(\mathcal{W},-)$-exact since $\mathcal{W} \perp \mathcal{X}$. Then

$$
\widehat{\operatorname{Ext}}_{\mathcal{W A}}^{0}(M, M) \cong \widehat{\operatorname{Ext}}_{\mathcal{W A}}^{n}\left(M_{-n}, M\right)=0
$$

by $[9,(4.6)]$, since $M_{-n} \in \operatorname{res} \widehat{X}$ and $\widehat{\operatorname{Ext}}_{W \mathcal{A}}^{t}\left(W_{i}, M\right)=0$ for any $t \in \mathbb{Z}$ and any $i \leq-1$ by Lemma 3.1.

(4) $\Rightarrow$ (1) holds by Lemma 3.2 and $[9,(3.4)]$.

Similarly, we can prove $(1) \Rightarrow\left(2^{\prime}\right) \Rightarrow\left(3^{\prime}\right) \Rightarrow(4)$.

The next corollary encompasses $[9,(5.6)$ and (5.7)] by noting that if $\mathcal{W}$ is closed under kernels of epimorphisms and $\mathcal{W} \perp \mathcal{W}$ then $\operatorname{res} \widehat{G(\mathcal{W})}=\operatorname{res} \overline{\mathcal{W}}$ (see $[9,(3.6)]$ ). The equivalence of $(1),\left(2^{\prime}\right)$ and $\left(3^{\prime}\right)$ of the following result was proved in $[9,(5.6)]$ by using $[9,(5.2)]$. However, we see that $[9,(5.2)]$ is in the special case when $\mathcal{X}=\mathcal{G}(\mathcal{W})$. Now we can prove it using Theorem 3.5.

Corollary 3.6. Assume that $\mathcal{X}$ is exact and closed under kernels of epimorphisms, and that $\mathcal{W}$ is both an injective cogenerator and a projective generator for $\mathcal{X}$ and closed under direct summands. Let $M \in \operatorname{res} \widehat{X}$ with $\mathcal{X}-\operatorname{pd}(M)=d<\infty$. Then the following statements are equivalent:

(1) $M \in \operatorname{res} \widehat{\mathcal{W}}$;

(2) The transformation $\vartheta_{X \mathcal{W A}}^{i}(-, M): \operatorname{Ext}_{\mathcal{X} \mathcal{A}}^{i}(-, M) \rightarrow \operatorname{Ext}_{\mathcal{W A}}^{i}(-, M)$ is an isomorphism on res $\widehat{X}$ for each $i \in \mathbb{Z}$;

$\left(2^{\prime}\right)$ The transformation $\vartheta_{\mathcal{X W \mathcal { A }}}^{i}(M,-): \operatorname{Ext}_{\mathcal{X} \mathcal{A}}^{i}(M,-) \rightarrow \operatorname{Ext}_{\mathcal{W A}}^{i}(M,-)$ is an isomorphism for each $i \in \mathbb{Z}$;

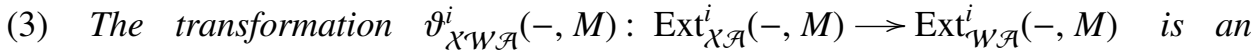
isomorphism on res $\widehat{X}$ for each $1 \leq i \leq 2$; 
(3') The transformation $\vartheta_{\mathcal{X} \mathcal{A}}^{i}(M,-): \operatorname{Ext}_{\mathcal{X} \mathcal{A}}^{i}(M,-) \rightarrow \operatorname{Ext}_{\mathcal{W A}}^{i}(M,-)$ is an isomorphism either for two successive values of $i$ with $1 \leq i<d$ or for a single value of $i$ with $i \geq d$;

Proof. $(1) \Leftrightarrow\left(2^{\prime}\right) \Leftrightarrow\left(3^{\prime}\right)$ can be proved as in the proof of $[9,(5.6)]$ using Theorem 3.5.

$(1) \Rightarrow$ (2) follows from $[8,(4.10)]$ since res $\widehat{X} \subseteq$ res $\widetilde{W} \cap \operatorname{res} \widetilde{X}$ by Lemma 2.6.

$(2) \Rightarrow(3)$ is trivial.

(3) $\Rightarrow(1)$. Let $N \in \operatorname{res} \widehat{\mathcal{X}}$, and let $\mathcal{X}-\operatorname{pd}(N)=t<\infty$. If $t=0$, then $N \in \mathcal{X}$, and so $\operatorname{Ext}_{\mathcal{X}}^{1}(N, M)=0$. Thus

$$
\operatorname{Ext}_{\mathcal{W A}}^{1}(N, M) \cong \operatorname{Ext}_{\mathcal{X} \mathcal{A}}^{1}(N, M)=0 .
$$

This implies that $\widehat{\operatorname{Ext}}_{\mathcal{W A}}^{1}(N, M)=0$ by [9, (4.10)]. Let $t=1$. Since $\vartheta_{\mathcal{X} \mathcal{W A}}^{1}(N, M)$ is an isomorphism, we get $\widehat{\operatorname{Ext}}_{\mathcal{W A}}^{1}(N, M)=0$ by [9, (4.10)]. Let $t \geq 2$. Since $\vartheta_{\mathcal{X} \mathcal{W} \mathcal{A}}^{1}(N, M)$ and $\vartheta_{\mathcal{X} \mathcal{W} \mathcal{A}}^{2}(N, M)$ are isomorphisms, we get that $\widehat{\operatorname{Ext}}_{\mathcal{W A}}^{1}(N, M)=0$ by [9, (4.10)]. Therefore, $\widehat{\operatorname{Ext}}_{\mathcal{W A}}^{1}(-, M)=0$ on res $\widehat{X}$, and so $M \in \operatorname{res} \widehat{\mathcal{W}}$ by Theorem 3.5.

The proofs of the next two results are dual to the previous two.

THEOREM 3.7. Assume that $\boldsymbol{Y}$ is exact and closed under cokernels of monomorphisms, and that $\mathcal{V}$ is both an injective cogenerator and a projective generator for $\mathcal{Y}$ and closed under direct summands. Let $N \in \operatorname{cores} \widehat{\mathcal{y}}$. Then the following statements are equivalent:

(1) $N \in \operatorname{cores} \widehat{\mathcal{V}}$;

(2) $\widehat{\operatorname{Ext}}_{\mathcal{A} \mathcal{V}}^{n}(N,-)=0$ on cores $\widehat{\mathcal{Y}}$ for each $n \in \mathbb{Z}$;

(2') $\widehat{\operatorname{Ext}}_{\mathcal{A V}}^{n}(-, N)=0$ for each $n \in \mathbb{Z}$;

(3) $\widehat{\operatorname{Ext}}_{\mathcal{A V}}^{n}(N,-)=0$ on cores $\widehat{y}$ for some $n \in \mathbb{Z}$;

(3') $\widehat{\operatorname{Ext}}_{\mathcal{H V}}^{n}(-, N)=0$ for some $n \in \mathbb{Z}$;

(4) $\widehat{\operatorname{Ext}}_{\mathcal{A V} \mathcal{V}}^{0}(N, N)=0$.

Corollary 3.8. Assume that $\mathcal{Y}$ is exact and closed under cokernels of monomorphisms, and that $\mathcal{V}$ is both an injective cogenerator and a projective generator for $\mathcal{Y}$ and closed under direct summands. Let $N \in \operatorname{cores} \widehat{\mathcal{Y}}$ with $\mathcal{Y}-\operatorname{id}(N)=d<\infty$. Then the following statements are equivalent.

(1) $N \in \operatorname{cores} \widehat{\mathcal{V}}$.

(2) The transformation $\vartheta_{\mathcal{A V} \mathcal{y}}^{i}(N,-): \operatorname{Ext}_{\mathcal{A} \mathcal{Y}}^{i}(N,-) \rightarrow \operatorname{Ext}_{\mathcal{A V}}^{i}(N,-)$ is an isomorphism on cores $\widehat{\mathcal{Y}}$ for each $i \in \mathbb{Z}$.

$\left(2^{\prime}\right)$ The transformation $\vartheta_{\mathcal{A V} y}^{i}(-, N): \operatorname{Ext}_{\mathcal{A Y}}^{i}(-, N) \rightarrow \operatorname{Ext}_{\mathcal{A V}}^{i}(-, N)$ is an isomorphism for each $i \in \mathbb{Z}$.

(3) The transformation $\vartheta_{\mathcal{A V} \mathcal{Y}}^{i}(N,-): \operatorname{Ext}_{\mathcal{A Y}}^{i}(N,-) \rightarrow \operatorname{Ext}_{\mathcal{A V}}^{i}(N,-)$ is an isomorphism on cores $\widehat{y}$ for each $1 \leq i \leq 2$. 
$\left(3^{\prime}\right)$ The transformation $\vartheta_{\mathcal{A V} y}^{i}(-, N): \operatorname{Ext}_{\mathcal{A Y}}^{i}(-, N) \rightarrow \operatorname{Ext}_{\mathcal{A V} V}^{i}(-, N)$ is an isomorphism either for two successive values of $i$ with $1 \leq i<d$ or for a single value of $i$ with $i \geq d$.

The next theorem is the main result of this paper, which was proved by SatherWagastaff et al. in the special case when $\mathcal{X}=\mathcal{G}(\mathcal{W}), \mathcal{Y}=\mathcal{G}(\mathcal{V})$ and $n \geq 1$ (see [9, (6.1)]).

Theorem 3.9. Assume that $\mathcal{X}$ and $\mathcal{Y}$ are exact, and $\mathcal{X}$ is closed under kernels of epimorphisms and $\mathcal{Y}$ is closed under cokernels of monomorphisms. Assume that $\mathcal{W}$ is both an injective cogenerator and a projective generator for $\mathcal{X}$ and $\mathcal{V}$ is both an injective cogenerator and a projective generator for $\mathcal{Y}$. Assume also that $\mathcal{W}$ and $\mathcal{V}$ are closed under direct summands and satisfy $\mathcal{W} \perp \mathcal{Y}, \mathcal{V} \perp \mathcal{V}$ and $\operatorname{Ext}_{\mathcal{W} \mathcal{A}}^{\geq 1}(\operatorname{res} \widehat{\mathcal{W}}, \mathcal{V})=0=\operatorname{Ext}_{\mathcal{A V} \mathcal{V}}^{\geq 1}(\mathcal{W}$, cores $\widehat{\mathcal{V}})$. Then, for all $M \in \operatorname{res} \widehat{\mathcal{X}}$ and $N \in \operatorname{cores} \widehat{\mathcal{Y}}$, and all $n \in \mathbb{Z}$,

$$
\widehat{\operatorname{Ext}}_{W \mathcal{A}}^{n}(M, N) \cong \widehat{\operatorname{Ext}}_{\mathcal{A V}}^{n}(M, N) \text {. }
$$

Proof. We first prove the case when $n \geq 1$ using a method similar to that of $[9,(6.1)]$. We give the proof here for the sake of completeness.

Note that $M \in \operatorname{res} \widehat{\mathcal{X}}$, so there is a Tate $\mathcal{W}$-resolution $T \stackrel{\alpha}{\rightarrow} W \rightarrow M$ of $M$ such that each $\operatorname{Coker}\left(\delta_{i}^{T}\right)$ is in $\mathcal{X}$ and each $\alpha_{i}$ is a split surjection for $i \in \mathbb{Z}$ by [9, (3.4)]. Thus there exists a degree-wise split exact sequence

$$
0 \rightarrow \Sigma^{-1} X \rightarrow \widetilde{T} \rightarrow W \rightarrow 0
$$

of complexes by [9, (3.10)], where $\widetilde{T}=T_{\supset-1}$ is exact with $\widetilde{T}_{-1} \in \mathcal{X}$, and $X$ is a bounded strict $\mathcal{W} \mathcal{X}$-resolution of $M$. Then, for $n \geq 1$,

$$
\widehat{\operatorname{Ext}}_{\mathcal{W A}}^{n}(M, N)=\mathrm{H}_{-n}\left(\operatorname{Hom}_{\mathcal{A}}(T, N)\right)=\mathrm{H}_{-n}\left(\operatorname{Hom}_{\mathcal{A}}(\widetilde{T}, N)\right) .
$$

Similarly, let $N \rightarrow V \stackrel{\beta}{\longrightarrow} S$ be a Tate $\mathcal{V}$-resolution of $N$ such that each $\operatorname{Ker}\left(\delta_{i}^{S}\right)$ is in $\mathcal{Y}$ and each $\beta_{i}$ is a split injection for $i \in \mathbb{Z}$. Then there exists a degree-wise split exact sequence

$$
0 \rightarrow V \rightarrow \widetilde{S} \rightarrow \Sigma Y \rightarrow 0
$$

of complexes by [9, (3.11)], where $\widetilde{S}=S_{\subset 1}$ is exact with $\widetilde{S}_{1} \in \mathcal{Y}$, and $Y$ is a bounded strict $\mathcal{Y} \mathcal{V}$-coresolution of $N$. Thus, for $n \geq 1$,

$$
\widehat{\operatorname{Ext}}_{\mathcal{A V} V}^{n}(M, N)=\mathrm{H}_{-n}\left(\operatorname{Hom}_{\mathcal{A}}(M, S)\right)=\mathrm{H}_{-n}\left(\operatorname{Hom}_{\mathcal{A}}(M, \widetilde{S})\right) \text {. }
$$

In the following, we show that $\mathrm{H}_{i}\left(\operatorname{Hom}_{\mathcal{A}}(\widetilde{T}, N)\right) \cong \mathrm{H}_{i}\left(\operatorname{Hom}_{\mathcal{A}}(M, \widetilde{S})\right)$ for any $i \in \mathbb{Z}$.

Note that $\widetilde{S}$ is an exact bounded above complex of objects in $\mathcal{Y}$, $\operatorname{so~}_{\mathcal{H}}\left(W_{i}, \tilde{S}\right)$ is exact for each $i$ since $\mathcal{W} \perp \mathcal{Y}$, and hence $\operatorname{Hom}_{\mathcal{A}}(W, \widetilde{S})$ is exact by [2, (2.4)]. Now consider the exact sequence

$$
0 \rightarrow \operatorname{Hom}_{\mathcal{A}}(W, \widetilde{S}) \rightarrow \operatorname{Hom}_{\mathcal{A}}(\widetilde{T}, \widetilde{S}) \rightarrow \operatorname{Hom}_{\mathcal{A}}\left(\Sigma^{-1} X, \widetilde{S}\right) \rightarrow 0
$$


then we get that $\operatorname{Hom}_{\mathcal{A}}(\widetilde{T}, \widetilde{S}) \rightarrow \operatorname{Hom}_{\mathcal{A}}\left(\Sigma^{-1} X, \widetilde{S}\right)$ is a quasiisomorphism. On the other hand, notice that $X$ is a bounded strict $\mathcal{W} X$-resolution of $M$, so $X$ is a proper $\mathcal{X}$-resolution of $M$. Thus the morphism

$$
\operatorname{Hom}_{\mathcal{A}}(M, \widetilde{S}) \rightarrow \operatorname{Hom}_{\mathcal{A}}(X, \widetilde{S})
$$

is a quasiisomorphism by $[8,(6.6)]$. Therefore, for any $i \in \mathbb{Z}$,

$$
\begin{aligned}
\mathrm{H}_{i}\left(\operatorname{Hom}_{\mathcal{A}}(M, \widetilde{S})\right) & \cong \mathrm{H}_{i}\left(\operatorname{Hom}_{\mathcal{A}}(X, \widetilde{S})\right) \\
& \cong \mathrm{H}_{i+1}\left(\operatorname{Hom}_{\mathcal{A}}\left(\Sigma^{-1} X, \widetilde{S}\right)\right) \\
& \cong \mathrm{H}_{i+1}\left(\operatorname{Hom}_{\mathcal{A}}(\widetilde{T}, \widetilde{S})\right) .
\end{aligned}
$$

Similarly, we get that $\mathrm{H}_{i}\left(\operatorname{Hom}_{\mathcal{A}}(\widetilde{T}, N)\right) \cong \mathrm{H}_{i+1}\left(\operatorname{Hom}_{\mathcal{A}}(\widetilde{T}, \widetilde{S})\right)$ for any $i \in \mathbb{Z}$. This implies that $\mathrm{H}_{i}\left(\operatorname{Hom}_{\mathcal{A}}(\widetilde{T}, N)\right) \cong \mathrm{H}_{i}\left(\operatorname{Hom}_{\mathcal{A}}(M, \widetilde{S})\right)$ for any $i \in \mathbb{Z}$. Thus, for $n \geq 1$,

$$
\widehat{\operatorname{Ext}}_{\mathcal{W A}}^{n}(M, N) \cong \widehat{\operatorname{Ext}}_{\mathcal{A V}}^{n}(M, N) \text {. }
$$

Now let $n=-d$ with $d \geq 0$, and we will prove that $\widehat{\operatorname{Ext}}_{\mathcal{W A}}^{n}(M, N) \cong \widehat{\operatorname{Ext}}_{\mathcal{A V} V}^{n}(M, N)$.

Since $M \in \operatorname{res} \widehat{\mathcal{X}}$, there is an exact sequence

$$
0 \longrightarrow M \longrightarrow W_{-1} \rightarrow M_{-1} \longrightarrow 0
$$

with $W_{-1} \in \operatorname{res} \widehat{\mathcal{W}}$ and $M_{-1} \in \mathcal{X}$ by [8, (3.3)]. Note that $\mathcal{W} \perp \mathcal{X}$, then $\mathcal{W} \perp$ res $\widehat{X}$, and so the sequence (II) is $\operatorname{Hom}_{\mathcal{A}}(\mathcal{W},-)$-exact. Since $\mathcal{W}$ is an injective cogenerator for $\mathcal{X}$, we get an exact sequence

$$
0 \rightarrow M_{-1} \rightarrow W_{-2} \rightarrow W_{-3} \rightarrow \cdots
$$

with each $W_{i} \in \mathcal{W}$ for $i \leq-2$, such that $M_{-i}=\operatorname{Im}\left(W_{-i} \rightarrow W_{-i-1}\right) \in \mathcal{X}$ for $i \geq 2$. Thus the sequence (IIII) is $\operatorname{Hom}_{\mathcal{A}}(\mathcal{W},-)$-exact. Notice that $\widehat{\operatorname{Ext}}_{\mathcal{W A}}^{j}\left(W_{-s}, A\right)=0$ for any object $A$ of $\mathcal{A}$, any $s \geq 1$ and any $j \in \mathbb{Z}$ by Lemma 3.1, and hence

$$
\widehat{\operatorname{Ext}}_{\mathcal{W A}}^{i}(M, A) \cong \widehat{\operatorname{Ext}}_{\mathcal{W A}}^{i+k}\left(M_{-k}, A\right)
$$

for any $k \geq 1$ and $i \in \mathbb{Z}$ by $[9,(4.7)]$.

On the other hand, by $[8,(3.3)]$, there is an exact sequence

$$
0 \rightarrow N_{1} \rightarrow V_{1} \rightarrow N \rightarrow 0
$$

with $V_{1} \in \operatorname{cores} \widehat{\mathcal{V}}$ and $N_{1} \in \mathcal{Y}$. Note that $\mathcal{Y} \perp \mathcal{V}$, then cores $\widehat{\mathcal{Y}} \perp \mathcal{V}$, and so the sequence $(\dagger)$ is $\operatorname{Hom}_{\mathcal{A}}(-, \mathcal{V})$-exact. Since $\mathcal{V}$ is a projective generator for $\mathcal{Y}$, we get an exact sequence

$$
\cdots \rightarrow V_{3} \rightarrow V_{2} \rightarrow N_{1} \rightarrow 0
$$


with each $V_{i} \in \mathcal{V}$ for $i \geq 2$ such that $N_{i}=\operatorname{Im}\left(V_{i+1} \rightarrow V_{i}\right) \in \mathcal{Y}$ for $i \geq 2$. Thus the sequence ( $\ddagger)$ is $\operatorname{Hom}_{\mathcal{A}}(-, \mathcal{V})$-exact. Notice that $\widehat{\operatorname{Ext}}_{\mathcal{A V}}^{j}\left(B, V_{s}\right)=0$ for any object $B$ of $\mathcal{A}$, any $s \geq 1$ and any $j \in \mathbb{Z}$ by Lemma 3.1, and therefore

$$
\widehat{\operatorname{Ext}}_{\mathcal{A} \mathcal{V}}^{i}(B, N) \cong \widehat{\operatorname{Ext}}_{\mathcal{A V} V}^{i+k}\left(B, N_{k}\right)
$$

for any $k \geq 1$ and $i \in \mathbb{Z}$ by [9, (4.7)].

Now we get the following isomorphisms:

$$
\begin{aligned}
\widehat{\operatorname{Ext}}_{W \mathcal{A}}^{-d}(M, N) & \cong \widehat{\operatorname{Ext}}_{\mathcal{W} \mathcal{A}}^{1}\left(M_{-d-1}, N\right) \\
& \cong \widehat{\operatorname{Ext}}_{\mathcal{A} \mathcal{V}}^{1}\left(M_{-d-1}, N\right) \\
& \cong \widehat{\operatorname{Ext}}_{\mathcal{A} \mathcal{V}}^{d+2}\left(M_{-d-1}, N_{d+1}\right) \\
& \cong \widehat{\operatorname{Ext}}_{\mathcal{W} \mathcal{A}}^{d+2}\left(M_{-d-1}, N_{d+1}\right) \\
& \cong \widehat{\operatorname{Ext}}_{\mathcal{W} \mathcal{A}}\left(M, N_{d+1}\right) \\
& \cong \widehat{\operatorname{Ext}}_{\mathcal{A V} \mathcal{V}}^{1}\left(M, N_{d+1}\right) \\
& \cong \widehat{\operatorname{Ext}}_{\mathcal{A} \mathcal{V}}(M, N),
\end{aligned}
$$

where the first and the fifth isomorphisms follow from (§), the third and the seventh hold by $(\S \S)$, and the remaining ones follow from $(\natural)$ since $d \geq 0$. Thus we get that $\widehat{\operatorname{Ext}}_{\mathcal{W A}}^{n}(M, N) \cong \widehat{\operatorname{Ext}}_{\mathcal{A V} \mathcal{V}}^{n}(M, N)$ for $n \leq 0$.

Therefore, we have $\widehat{\operatorname{Ext}}_{\mathcal{W A}}^{n}(M, N) \cong \widehat{\operatorname{Ext}}_{\mathcal{A V}}^{n}(M, N)$ for all $M \in \operatorname{res} \widehat{\mathcal{X}}$ and $N \in$ cores $\widehat{\mathcal{Y}}$, and all $n \in \mathbb{Z}$.

Corollary 3.10. Assume that $\mathcal{W} \perp \mathcal{W}, \mathcal{V} \perp \mathcal{V}, \mathcal{G}(\mathcal{W}) \perp \mathcal{V}$ and $\mathcal{W} \perp \mathcal{G}(\mathcal{V})$. Assume that $\mathcal{W}$ is closed under kernels of epimorphisms and direct summands and that $\mathcal{V}$ is closed under cokernels of monomorphisms and direct summands. Assume also that $\operatorname{Ext}_{\mathcal{W} \mathcal{A}}^{\geq 1}(\operatorname{res} \widehat{\mathcal{W}}, \mathcal{V})=0=\operatorname{Ext}_{\mathcal{A V} \mathcal{V}}^{\geq 1}(\mathcal{W}$, cores $\widehat{\mathcal{V}})$. Then, for all $\left.M \in \operatorname{res} \widehat{\mathcal{G}(\widehat{W}}\right)$, all $N \in \operatorname{cores} \widehat{\mathcal{G}(\mathcal{V})}$ and all $n \in \mathbb{Z}$,

$$
\widehat{\operatorname{Ext}}_{\mathcal{W A}}^{n}(M, N) \cong \widehat{\operatorname{Ext}}_{\mathcal{A V} \mathcal{V}}^{n}(M, N) .
$$

Proof. Immediately by Theorem 3.9 and Remark 2.4.

We write $\mathcal{P}$ and $\mathcal{I}$ for the subcategories of projective left $R$-modules and injective left $R$-modules, respectively. One can check easily that $\mathcal{W}=\mathcal{P}$ and $\mathcal{V}=\mathcal{I}$ satisfy the hypotheses of Corollary 3.10, thus we have the next corollary that can be found in [3, Theorem 5.4] and [4, Corollary 3.4].

Corollary 3.11. Let $M$ and $N$ be left $R$-modules such that

$$
\mathcal{G}(\mathcal{P})-\operatorname{pd}_{R}(M)<\infty \quad \text { and } \quad \mathcal{G}(\mathcal{I})-\mathrm{id}_{R}(N)<\infty .
$$


Then, for each $n \in \mathbb{Z}$,

$$
\widehat{\operatorname{Ext}}_{R}^{n}(M, N)=\widehat{\operatorname{Ext}}_{\mathcal{P} \mathcal{M}}^{n}(M, N) \cong \widehat{\operatorname{Ext}}_{\mathcal{M} I}^{n}(M, N) .
$$

Let $R$ be a commutative ring. An $R$-module $C$ is called semidualizing if $C$ admits a degree-wise finite projective resolution, $\operatorname{Ext}_{R}^{\geq 1}(C, C)=0$ and the natural homothety map $R \rightarrow \operatorname{Hom}_{R}(C, C)$ is an isomorphism. Examples include the rankone free $R$-modules and a dualizing (canonical) $R$-module (when one exists). We let $\mathcal{P}_{C}$ (respectively, $\mathcal{I}_{C}$ ) denote the subcategory of $R$-modules $C \otimes_{R} P$ (respectively, $\operatorname{Hom}_{R}(C, I)$ ) with $P$ (respectively, $\left.I\right)$ projective (respectively, injective). Modules in $\mathcal{P}_{C}$ and $\mathcal{I}_{C}$ are called $C$-projective and $C$-injective, respectively. A complete $\mathcal{P P}_{C^{-}}$ resolution is an exact and $\operatorname{Hom}_{R}\left(-, \mathcal{P}_{C}(R)\right.$ )-exact complex $X$ of $R$-modules with $X_{i}$ projective for $i \geq 0$ and $X_{j} C$-projective for $j<0$. An $R$-module $M$ is $\mathrm{G}_{C}$-projective if there exists a complete $\mathcal{P} \mathcal{P}_{C}$-resolution $X$ such that $M \cong \operatorname{Ker}\left(\delta_{-1}^{X}\right)$. We let $\mathcal{G P}_{C}(R)$ denote the subcategory of $\mathrm{G}_{C}$-projective $R$-modules.

Let $B$ and $C$ be semidualizing $R$-modules such that $B \in G \mathcal{P}_{C}(R)$. Set $B^{\dagger}=$ $\operatorname{Hom}_{R}(B, C)$, then $B^{\dagger}$ is a semidualizing $R$-module. Now $\mathcal{W}=\mathcal{P}_{B}(R)$ and $\mathcal{V}=\mathcal{I}_{B^{\dagger}}(R)$ satisfy the hypotheses of Corollary 3.10 by the proof of $[9,(6.2)]$. Thus we have the next result that was proved by Sather-Wagstaff et al. for $n \geq 1$ (see [9, Theorem D]).

Corollary 3.12. Let $R$ be a commutative ring, and let $B$ and $C$ be semidualizing $R$ modules such that $B \in G^{P} P_{C}(R)$. Set $B^{\dagger}=\operatorname{Hom}_{R}(B, C)$. Let $M$ and $N$ be R-modules such that

$$
\mathcal{G}\left(\mathcal{P}_{B}\right)-\operatorname{pd}_{R}(M)<\infty \quad \text { and } \quad \mathcal{G}\left(\mathcal{I}_{B^{\dagger}}\right)-\mathrm{id}_{R}(N)<\infty .
$$

Then, for each $n \in \mathbb{Z}$,

$$
\widehat{\operatorname{Ext}}_{\mathcal{P}_{B} \mathcal{M}}^{n}(M, N) \cong \widehat{\operatorname{Ext}}_{\mathcal{M} I_{B^{\dagger}}}^{n}(M, N)
$$

In the following, we let $\mathcal{X}, \mathcal{Y}, \mathcal{W}$ and $\mathcal{V}$ denote subcategories of $\mathcal{M}$ (the category of left $R$-modules).

Assume that $\mathcal{X}$ is closed under extensions, and $\mathcal{W}$ is both an injective cogenerator and a projective generator for $\mathcal{X}$. Let $M \in \operatorname{res} \widehat{\mathcal{X}}$. Then $M$ has a proper $\mathcal{X}$-resolution $X \stackrel{\simeq}{\rightarrow} M$ and a proper $\mathcal{W}$-resolution $W \stackrel{\simeq}{\longrightarrow} M$ by Lemma 2.6. Set $_{\overline{\mathrm{id}}} M: W \rightarrow X$ a lifting of the identity $\operatorname{id}_{M}: M \rightarrow M$. Then we have the following result that provides a new method to compute Tate cohomology.

Proposition 3.13. Assume that $\mathcal{X}$ is exact and closed under kernels of epimorphisms, and $\mathcal{W}$ is both an injective cogenerator and a projective generator for $\mathcal{X}$ and closed under direct summands. Let $M \in \operatorname{res} \widehat{\mathcal{X}}$. Then

$$
\widehat{\operatorname{Ext}}_{W \mathcal{M}}^{n}(M, N) \cong \mathrm{H}_{-n-1}\left(\operatorname{Hom}_{R}\left(\operatorname{Cone}\left(\overline{\operatorname{id}_{M}}\right), N\right)\right)
$$

for any left $R$-module $N$ and any $n \geq 1$. 
Proof. By [9, (3.4)], there is a Tate $\mathcal{W}$-resolution $T \stackrel{\alpha}{\rightarrow} W \stackrel{\eta}{\rightarrow} M$ of $M$ such that $\operatorname{Coker}\left(\delta_{1}^{T}\right) \in \mathcal{X}$ and $\alpha_{n}$ are split surjections for all $n \in \mathbb{Z}$. Using [9, (3.10)] we get a degree-wise split exact sequence

$$
0 \rightarrow \Sigma^{-1} X \stackrel{\lambda}{\rightarrow} \widetilde{T} \stackrel{\alpha}{\rightarrow} W \rightarrow 0
$$

of complexes with $\widetilde{T}$ exact, where $X \stackrel{\simeq}{\longrightarrow} M$ is a bounded strict $\mathcal{W} X$-resolution of $M$ and $W \stackrel{\simeq}{\longrightarrow} M$ is a proper $\mathcal{W}$-resolution of $M$. Since $X \perp \mathcal{W}$, we get that $X \stackrel{\simeq}{\longrightarrow} M$ is a proper $\mathcal{X}$-resolution of $M$. By the proof of [9, (3.10)], we can rewrite the sequence (II) as follows.

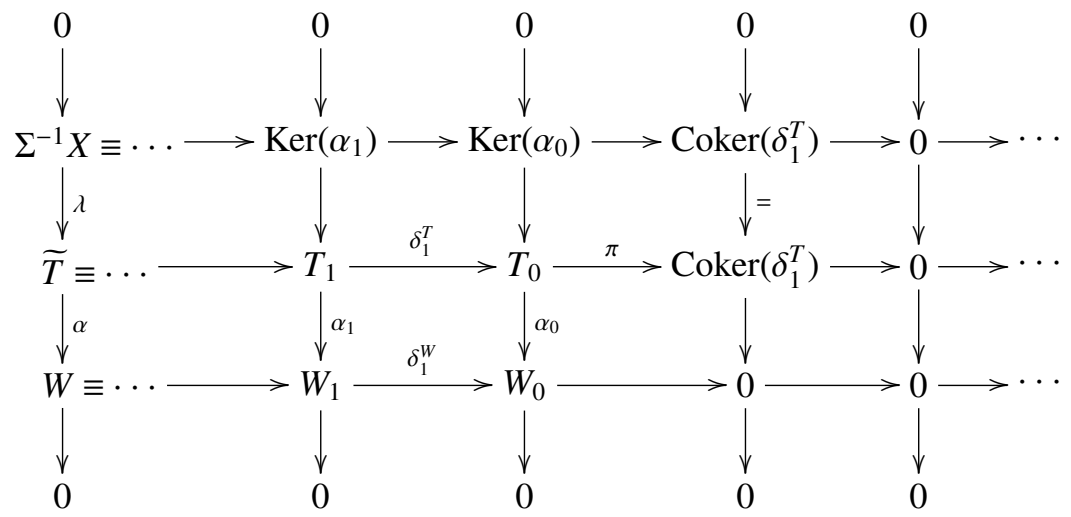

Since the sequence $(\|)$ is degree-wise split, there is $\alpha_{i}^{\prime}: W_{i} \rightarrow T_{i}$ for $i \geq 0$ such that $\alpha_{i} \alpha_{i}^{\prime}=\mathrm{id}_{W_{i}}$. Thus we get the following commutative diagram

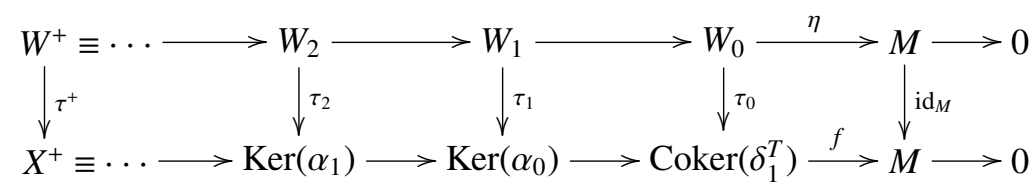

with the first row an augmented proper $\mathcal{W}$-resolution of $M$ and the second row an augmented proper $\mathcal{X}$-resolution of $M$, where $\tau_{0}=\pi \alpha_{0}^{\prime}$ and $\tau_{i}=(-1)^{i}\left(\delta_{i}^{T} \alpha_{i}^{\prime}-\alpha_{i-1}^{\prime} \delta_{i}^{W}\right)$ for $i \geq 1$, and $f\left(x+\operatorname{Im} \delta_{1}^{T}\right)=\eta \alpha_{0}(x)$ for any $x \in T_{0}$. Now one can check that $\widetilde{T} \cong$ $\Sigma^{-1} \operatorname{Cone}(\tau)$. Thus, for $n \geq 1$,

$$
\begin{aligned}
\widehat{\operatorname{Ext}}_{W \mathcal{M}}^{n}(M, N) & =\mathrm{H}_{-n}\left(\operatorname{Hom}_{R}(T, N)\right) \\
& \cong \mathrm{H}_{-n}\left(\operatorname{Hom}_{R}(\widetilde{T}, N)\right) \\
& \cong \mathrm{H}_{-n}\left(\operatorname{Hom}_{R}\left(\Sigma^{-1} \operatorname{Cone}(\tau), N\right)\right) \\
& \cong \mathrm{H}_{-n-1}\left(\operatorname{Hom}_{R}(\operatorname{Cone}(\tau), N)\right) \\
& \cong \mathrm{H}_{-n-1}\left(\operatorname{Hom}_{R}\left(\operatorname{Cone}\left(\overline{\operatorname{id}_{M}}\right), N\right)\right),
\end{aligned}
$$


where the second isomorphism holds since $\widetilde{T}=T_{\supset-1}$, and the last isomorphism follows from the fact that $\operatorname{Cone}\left(\overline{\mathrm{id}_{M}}\right)$ and $\operatorname{Cone}(\tau)$ are homotopy equivalent [6, page 392].

The next result is proved dually by noting that if $\mathcal{Y}$ is closed under extensions and $\mathcal{V}$ is both an injective cogenerator and a projective generator for $\mathcal{Y}$, then cores $\widehat{\mathcal{Y}} \subseteq$ cores $\widetilde{\mathcal{V}} \cap \operatorname{cores} \widetilde{\mathcal{Y}}$ by Lemma 2.6.

Proposition 3.14. Assume that $\mathcal{Y}$ is exact and closed under cokernels of monomorphisms, and $\mathcal{V}$ is both an injective cogenerator and a projective generator for $\mathcal{Y}$ and closed under direct summands. Let $N \in \operatorname{cores} \widehat{\mathcal{Y}}$, and let $N \stackrel{\simeq}{\rightarrow} Y$ be $a$ proper $\mathcal{Y}$-coresolution and $N \stackrel{\simeq}{\rightarrow} V$ a proper $\mathcal{V}$-coresolution of $N$, and let $\overline{\mathrm{id}_{N}}$ : $Y \rightarrow V$ be a lifting of the identity $\operatorname{id}_{N}: N \rightarrow N$. Then

$$
\widehat{\operatorname{Ext}}_{\mathcal{M V}}^{n}(M, N) \cong \mathrm{H}_{-n}\left(\operatorname{Hom}_{R}\left(M, \text { Cone }\left(\overline{\mathrm{id}_{N}}\right)\right)\right)
$$

for any left $R$-module $M$ and any $n \geq 1$.

The next corollary is immediate by Theorem 3.9 and Propositions 3.13 and 3.14.

Corollary 3.15. Assume that $\mathcal{X}$ and $\mathcal{Y}$ are exact, $\mathcal{X}$ is closed under kernels of epimorphisms and $\mathcal{Y}$ is closed under cokernels of monomorphisms. Assume that $\mathcal{W}$ is both an injective cogenerator and a projective generator for $\mathcal{X}$, and $\mathcal{V}$ is both an injective cogenerator and a projective generator for $\mathcal{Y}$. Assume also that $\mathcal{W}$ and $\mathcal{V}$ are closed under direct summands and satisfy $\mathcal{W} \perp \mathcal{Y}, \mathcal{X} \perp \mathcal{V}$ and $\operatorname{Ext}_{\mathcal{W} \mathcal{A}}^{\geq 1}(\operatorname{res} \widehat{\mathcal{W}}, \mathcal{V})=0=\operatorname{Ext}_{\mathcal{A V} \mathcal{V}}^{\geq 1}(\mathcal{W}$, cores $\widehat{\mathcal{V}})$. Then, for all $M \in \operatorname{res} \widehat{\mathcal{X}}$ and $N \in \operatorname{cores} \widehat{\mathcal{Y}}$, and all $n \geq 1$,

$$
\begin{aligned}
\widehat{\operatorname{Ext}}_{W \mathcal{A}}^{n}(M, N) & \cong \widehat{\operatorname{Ext}}_{\mathcal{A V}}^{n}(M, N) \\
& \cong \mathrm{H}_{-n-1}\left(\operatorname{Hom}_{R}\left(\operatorname{Cone}\left(\overline{\operatorname{id}_{M}}\right), N\right)\right) \\
& \cong \mathrm{H}_{-n}\left(\operatorname{Hom}_{R}\left(M, \operatorname{Cone}\left(\overline{\operatorname{id}_{N}}\right)\right)\right)
\end{aligned}
$$

Let $R$ be a commutative ring, and let $B$ and $C$ be semidualizing $R$-modules such that $B \in G \mathcal{P}_{C}(R)$. Set $B^{\dagger}=\operatorname{Hom}_{R}(B, C)$. Let $M$ and $N$ be $R$-modules such that $\mathcal{G}\left(\mathcal{P}_{B}\right)-\operatorname{pd}_{R}(M)<\infty$ and $\mathcal{G}\left(\mathcal{I}_{B^{\dagger}}\right)-\mathrm{id}_{R}(N)<\infty$. Then $M$ has a proper $\mathcal{G}\left(\mathcal{P}_{B}\right)$-resolution $X \stackrel{\simeq}{\rightarrow} M$ and a proper $\mathcal{P}_{B}$-resolution $W \stackrel{\simeq}{\rightarrow} M$ by Lemma 2.6. Set $\overline{\mathrm{id}_{M}}: W \rightarrow X$ a lifting of the identity $\operatorname{id}_{M}: M \rightarrow M$. Dually, one can construct $\overline{\mathrm{id}_{N}}$. Then we have the next result by Corollary 3.15 .

Corollary 3.16. Let $R$ be a commutative ring, and let $B$ and $C$ be semidualizing $R$ modules such that $B \in G^{P} P_{C}(R)$. Set $B^{\dagger}=\operatorname{Hom}_{R}(B, C)$. Let $M$ and $N$ be R-modules such that

$$
\mathcal{G}\left(\mathcal{P}_{B}\right)-\operatorname{pd}_{R}(M)<\infty \quad \text { and } \quad \mathcal{G}\left(\mathcal{I}_{B^{\dagger}}\right)-\mathrm{id}_{R}(N)<\infty \text {. }
$$


Then, for each $n \geq 1$,

$$
\begin{aligned}
\widehat{\operatorname{Ext}}_{\mathcal{P}_{B} \mathcal{M}}(M, N) & \cong \widehat{\operatorname{Ext}}_{\mathcal{M} I_{B^{\dagger}}}^{n}(M, N) \\
& \cong \mathrm{H}_{-n-1}\left(\operatorname{Hom}_{R}\left(\operatorname{Cone}\left(\overline{\operatorname{id}_{M}}\right), N\right)\right) \\
& \cong \mathrm{H}_{-n}\left(\operatorname{Hom}_{R}\left(M, \operatorname{Cone}\left(\overline{\overline{\mathrm{id}}_{N}}\right)\right)\right)
\end{aligned}
$$

\title{
Acknowledgements
}

We thank Professor Nanqing Ding and Professor Zhongkui Liu for constant help and encouragement. We also thank the referee for the useful suggestions.

\section{References}

[1] L. L. Avramov and A. Martsinkovsky, 'Absolute, relative, and Tate cohomology of modules of finite Gorenstein dimension', Proc. Lond. Math. Soc. 85 (2002), 393-440.

[2] L. W. Christensen, A. Frankild and H. Holm, 'On Gorenstein projective, injective and flat dimensions - a functorial description with applications', J. Algebra 302 (2006), 231-279.

[3] L. W. Christensen and D. A. Jorgensen, 'Tate (co)homology via pinched complexes', Trans. Amer. Math. Soc.; doi:10.1090/S0002-9947-2013-05746-7.

[4] E. E. Enochs, S. Estrada and A. Iacob, 'Balance with unbounded complexes', Bull. Lond. Math. Soc. 44 (2012), 439-442.

[5] H. Holm, 'Gorenstein derived functors', Proc. Amer. Math. Soc. 132 (2004), 1913-1923.

[6] A. Iacob, 'Generalized Tate cohomology', Tsukuba J. Math. 29 (2005), 389-404.

[7] S. Sather-Wagstaff, T. Sharif and D. White, 'Stability of Gorenstein categories', J. Lond. Math. Soc. 77 (2008), 481-502.

[8] S. Sather-Wagstaff, T. Sharif and D. White, 'Gorenstein cohomology in abelian categories', J. Math. Kyoto Univ. 48 (2008), 571-596.

[9] S. Sather-Wagstaff, T. Sharif and D. White, 'Tate cohomology with respect to semidualizing modules', J. Algebra 324 (2010), 2336-2368.

\author{
LI LIANG, School of Mathematics and Physics, Lanzhou Jiaotong University, \\ Lanzhou 730070, PR China \\ e-mail: 1liangnju@gmail.com
}

GANG YANG, School of Mathematics and Physics, Lanzhou Jiaotong University,

Lanzhou 730070, PR China

e-mail: yanggang@mail.lzjtu.cn 